\title{
Research on Government Decision Support System Based on Large Data
}

\author{
Wen Qiuhua \\ Academy of Overseas Chinese Studies In Jinan University, Guangzhou, 510632,China \\ qiuhuawen80@sina.com
}

Key words: Data mining; decision support system

Abstract. Decision support system is widely used. This paper presents a comprehensive introduction to the decision information system, including the concept, structure, development, system type and application fields. Then, the decision support system based on data warehouse, on-line analysis and data mining is introduced.

\section{Introduction}

With the development of computer technology, computer technology can provide more comprehensive and in-depth, computer technology in data collection, collation, storage, analysis and other aspects have a strong advantage, people will naturally make decisions and computing machine technology, thus forming a new discipline - decision support system. Decision support system has been developed rapidly, and has been developed into a new, comprehensive, and all kinds of systems.

With China's accession to the world trade organization's success, the world economic globalization has made our country's telecommunication enterprise face the unprecedented challenge. Whether to make quick and scientific decision to the problem, the success or failure of the telecommunication enterprise has a great influence. At present, it is widely used by the telecom operators to rely on manual data analysis data, which has a lot of problems, such as heavy workload, slow speed, easy to lose data. Therefore, the research and development of decision support system has become the consensus of many telecom operators in China.

Today's society is an era of information explosion, how to make full use of all kinds of information for human services has become increasingly important. As a new technology of knowledge discovery, data mining and decision support system, decision support system has been paid more and more attention, which provides a convenient way for people to get interesting and useful information from a large amount of data. In the research of data mining, classification and prediction is a very important problem. Many scholars have done a lot of research from various perspectives such as statistics, information theory, neural network and so on, and have made a series of important results.

\section{Decision support system is simply introduced}

DSS (Support System Decision) is a kind of computer application system, which is based on management science, operations research, control theory and behavior science. The concept of decision support system has been proposed in 1970s, along with the continuous development of science and technology, decision support system and a variety of emerging technologies (such as artificial intelligence, data warehouse, data mining, data analysis, data processing, etc.), a variety of more effective decision support systems, such as intelligent decision support system, distributed decision support system, group decision support system, based on network decision support system, etc..

Decision support system is composed of five parts, which are the human machine interface, database, model base, knowledge base and method base.

Library is the basis of decision support system. Decision support system is a cross discipline, in addition to computer application system, management science, operations research, control and 
behavior science, he also and psychology, economics, artificial intelligence and other disciplines, the development of other disciplines to promote the development of decision support system. With the improvement and development of decision support system, its application is becoming wider and wider, which is widely used in the fields such as enterprise management decision, economic situation forecast, forecast of natural disasters, policy decision and so on.

Although DSS has made great progress in China, there are still many problems in theory and practice. Such as DSS application is characterized by multi level, small scale; overall development is slow, the software production cycle is long, the productivity is low, the quality is difficult to guarantee, the development and application of the DSS application development is less, especially in the manufacturing industry. On the basis of the above problems, this paper discusses the development of DSS (Decision Support System) theory, describes the development process of DSS system, summarizes the application of DSS, and prospects the application of DSS in the analysis of its advantages and disadvantages. This paper comprehensively studies and analyzes the status of enterprises, computer software and hardware configuration and management of the need for such factors, the paper puts forward the DSS design scheme based on the present situation of coal mining machine, and uses the appropriate method to design the entity, the application effect is good. Also accumulated valuable experience in system development.

\section{Decision support system based on data warehouse, on-line processing analysis and data mining}

\section{1 data warehouse}

Data warehouse is a space that is used to access the data, but it is not just a simple stack of data.

But in the continuous development of the system, which is similar to a spider web, data extraction is carried out. Using literature retrieval database as an example, a connected to a database of a large number of large database, stored in the library of we do not need the original, but literature titles to digest the information, by finding the titles to digest the information, if Abstract titles information with other literature database is connected, you can get the full text. This is one of the most commonly used relational database technology. In addition, there are other different types of database systems, such as multidimensional database technology. Each technique has its advantages and defects, such as relational database is suitable for developing data driven decision support system, and multidimensional database has better performance in relation analysis and processing.

Decision support system and its related technology is a hot topic in the field of information. Based on data warehouse, the data warehouse is based on the traditional database and the data warehouse is the main source of information. The data and information are analyzed and processed by the technology of on-line analytical processing and data mining.

3.2 on-line processing analysis

The database is built, then the data is analyzed and processed, and the on-line analytical processing is the most commonly used.

One technique. The data warehouse focuses on the storage of data, and the online analysis processing focuses on the analysis of the materials, to provide help for the decision-making. There are two most important concepts in the on-line analysis processing, which is "dimension" and "measure". In the on-line analytical processing, information is seen as a cube, in the analysis of information, of the cube of slicing, dicing, drilling and rotation of the different mode of operation, to extract data from different angles. For example, this product sales information will include the date, product number, warehouse number and other information, and every information will point to a different dimension table, date will point to date table (annual, quarter, month, day), product number will refer to the product dimension table (Department, type, brand, Description), warehouse number will point to warehouse (province, city, inventory, warehouse name).

Next we will introduce some on-line analytical processing operations:

(1) drill into roll up and drill down, roll up can get high on the one-dimensional information from lower dimensional information, such as 
Warehouse dimension tables in volumes can be sales dimension table information, in turn, drill can be get down one-dimensional information, such as warehouse dimension table drill can get warehouse name information.

cuts refers to in a certain range of the dimension endo take part of the property is a cube, is section in a dimension of certain attribute were harvested and obtained is a slice. Such as Shanghai and Beijing's income and consumption are harvested is diced, and will cut Beijing's income, consumption, residents happiness index taken out is sliced.

3.3 data mining

The purpose of data mining is to extract and analyze data, and data mining can be regarded as the higher of the on-line analytical processing.

Stage, because it is the support of the database, the use of knowledge and mining engine to more functions of data processing. To achieve this effect is because data mining application of a unique mining algorithm and knowledge, and combined with more knowledge such as artificial intelligence technology, information theory, statistical analysis, fuzzy theory, machine learning methods, and so on, so it can be extracted and analyzed on the basis of information. With the continuous development of data mining, it is also applied to other new development decision support systems, such as network (Web) decision support system.

Because there is a large amount of data in the database, it is very important to find valuable information from the database. Data mining technology is to solve this problem. The research of data mining technology has made many remarkable achievements in China and abroad, and has been successfully applied to many fields, but it is not widely used in the field of education. College entrance examination affects countless candidates and parents of the heart, is the focus of social concern. Every year, the college entrance examination after the end, large amounts of data related to archive for future reference for the purpose of preservation, year after year, a large amount of historical data contains the potential value of the yet to be opened. The current network and information process has changed people's lives, the candidates and parents have been unable to meet the results of the candidates, admission scores and other information on the surface of the surface, they hope to get personalized high quality information service. In this paper, data warehouse and data mining technology is analyzed to construct a new type of decision support system based on the comprehensive analysis of the historical data in the college entrance examination. In this paper, a data warehouse based on data warehouse is used to construct data warehouse, which is based on data warehouse. At the same time, through the research on the classification method of data mining technology, the decision-making module of the college entrance examination is realized. Classification is an important part of data mining. It establishes the model according to the known data. Then it uses the model to predict the class of unknown data. The common classification methods are decision tree classification, Bias classification, neural network classification, and the decision tree method is superior to other classification methods in the aspects of intelligibility, ease of training, ease of implementation and generality. In this paper, the decision tree classification method is applied to the research of the college entrance examination. According to the proposed scheme, this paper takes the analysis of voluntary reporting as an example, the whole process of data mining, including: Data Mining object and target acquisition data, data integration, data cleaning, data conversion, data reduction and other data preprocessing technology. Using ID3 decision tree algorithm to generate decision tree, and use the pruning method to prune decision tree. The establishment of the decision tree model of the college entrance examination. The conclusion shows that the decision support system based on data warehouse, on-line analytical processing and data mining has some practical significance and wide application prospect.

\section{Concluding remarks}

In this paper, we focus on the decision support system based on data warehouse, on-line process analysis and data mining, there are some traditional decision support system and the new developing decision support system, which can be applied to different professional fields, such as 
the establishment of network teaching platform in the field of education, to promote the exchange between teachers and students, and make students learn more convenient.

\section{Acknowledgements}

Supported by the following projects: overseas Chinese Affairs Office of the Guangdong Province project "overseas Chinese professionals in Guangdong innovation and entrepreneurship research" GDQW201418, Overseas Chinese talent recruitment promote Guangdong's economic transformation upgrade research -201207,Guangdong Province Science and technology plan project" Application of mobile Internet service provided technology research"- (2014A040401027) .

\section{References}

[1] Li Xudong, Wang Yan. Data mining technology in the intelligent decision system should be used [J]. Gansu Branch Technology Horizontal, 2013 (04): 19-21.

[2] Zheng Delong, Li Mingming. Application of decision support system based on data mining [J]. engineering control computer, 2013 (05): 119-121.

[3] Du Juan. High level decision support system in the data mining technology [J].Modern computer: 2011 (16): 20-23.

[4] Chen Xi Wang Zhiquan. Overview of [J]. control and decision making, 2006 (09): 961-968.

[5] Cao Xiaojing Zhang Hang. Development of decision support system and its key technology analysis [J]. computer technology and development, 2006 (11): 94-96.

[6] Liu Bo Yuan, Fan Wen Hui. Xiao Tian Yuan. Decision support system research and development of [J]. system simulation Newspaper, 2011 (S1): 241-244. 